\title{
0751 INJURY PREVENTION: DEVELOPMENT AND USE
}

D Hungerford, Z Quigg*, HEdwards, I Warren, K Hughes, M A Bellis Correspondence: Centre for Public Health Research Directorate, Faculty of Health and Applied Social Sciences, Liverpool John Moores University, Kingsway House, 5th Floor Hatton, Garden L3 2AJ, UK

10.1136/ip.2010.029215.751

Aim The Trauma and Injury Intelligence Group (TIIG) Injury Surveillance System (ISS) was established in the North West of England to improve injury data collection and sharing between public health professionals; focusing on Accident and Emergency Department (AED) data. Implementation of the ISS allows local agencies to understand the burden of injuries, target interventions towards at risk groups and communities, and evaluate injury prevention initiatives. This presentation discusses the development, use and sustainability of a population based ISS to prevent injury.

Methods Data sharing and dissemination protocols were developed that enabled data from AEDs and other agencies (eg, ambulance/fire service) to be collated, analysed and shared with partner agencies. With addressing issues such as falls, 
alcohol and violence becoming increasingly important among local partners, TIIG facilitates the development of AED systems to record further information on the location and circumstances of intentional and unintentional injuries.

Results Data have been used to develop, implement and evaluate interventions aimed at preventing injuries in children and older adults; inform violence prevention in nightlife settings; and to evaluate local injury campaigns. These initiatives have been associated with reductions in AED injury attendances. Barriers to project development and sustainability include: variations and changes in AED data collection systems; data quality issues and resource constraints.

Discussion The ISS is helping professionals prevent injury through targeting resources towards risk locations, groups and communities. Collecting and sharing data on intentional and unintentional injury ensures the TIIG ISS has the flexibility to adapt to emerging issues. 